\title{
Measurements of the absolute energy spectra of cosmic-ray positrons and electrons above $7 \mathrm{GeV}$
}

\author{
C. Grimani ${ }^{1, \star}$, S. A. Stephens ${ }^{7}$, F. S. Cafagna ${ }^{2}$, G. Basini ${ }^{6}$, R. Bellotti ${ }^{2}$, M. T. Brunetti ${ }^{1}$, M. Circella ${ }^{2}$, A. Codino ${ }^{1}$, \\ C. De Marzo ${ }^{2}$, M. P. De Pascale ${ }^{3}$, N. Finetti ${ }^{1, \star \star}$, R. L. Golden ${ }^{4, \star \star \star}$, M. Hof $^{5}$, W. Menn ${ }^{5}$, J. W. Mitchell ${ }^{7}$, \\ A. Morselli ${ }^{3}$, J. F. Ormes ${ }^{7}$, P. Papini ${ }^{8}$, C. Pfeifer ${ }^{5}$, S. Piccardi ${ }^{8}$, P. Picozza ${ }^{3}$, M. Ricci ${ }^{6}$, M. Simon ${ }^{5}$, P. Spillantini ${ }^{8}$, \\ S. J. Stochaj ${ }^{4}$, and R. E. Streitmatter ${ }^{7}$
}

1 INFN and Dipartimento di Fisica dell'Università di Perugia, Via Pascoli, Perugia 06100, Italy

2 INFN and Dipartimento di Fisica dell'Università di Bari, Via Amendola, Bari 70126, Italy

3 INFN and Dipartimento di Fisica dell'Università di Roma II, Via della Ricerca Scientifica, Roma 00133, Italy

${ }^{4}$ Particle Astrophysics Laboratory, New Mexico State University, Box 3PAL, Las Cruces, New Mexico 88003, USA

5 Universität Siegen, Siegen 57068, Germany

${ }^{6}$ Laboratori Nazionali di Frascati,Via E. Fermi, Frascati 00044, Italy

7 NASA/Goddard Space Flight Center, Code 661, Greenbelt, MD 20771, USA

8 INFN and Dipartimento di Fisica dell’Università di Firenze, Largo E. Fermi, Firenze 50125, Italy

Received 17 January 2002 / Accepted 13 May 2002

\begin{abstract}
A measurement of the energy spectra of cosmic-ray positrons and electrons was made with a balloon-borne magnetspectrometer, which was flown at a mean geomagnetic cut-off of $4.5 \mathrm{GV} / \mathrm{c}$. The observed positron flux in the energy range $7-16 \mathrm{GeV}$ is approximately an order of magnitude lower than that of electrons, as measured in other experiments at various energies. The power law spectral index of the observed differential energy spectrum of electrons is $-2.89 \pm 0.10$ in the energy interval 7.5-47 GeV. For positrons the overall fit of the available data above $7 \mathrm{GeV}$ has been considered. The spectral index is found to be $-3.37 \pm 0.26$ and the fraction of positrons, $\mathrm{e}^{+} /\left(\mathrm{e}^{+}+\mathrm{e}^{-}\right)$, has a mean value of $0.064 \pm 0.003$. The world data on $\mathrm{e}^{+} /\left(\mathrm{e}^{+}+\mathrm{e}^{-}\right)$from 0.1 to $30 \mathrm{GeV}$ indicate that a plerion type electron spectrum is preferred over the other types. The trend of the presently existing high energy data also suggests a possible contribution of positrons produced at the pulsar polar cap. High resolution experiments capable of identifying positrons at least up to $100 \mathrm{GeV}$ with high statistics are required to pinpoint the origin of both electrons and positrons in the cosmic radiation.
\end{abstract}

Key words. ISM: cosmic rays

\section{Introduction}

Since the first observation of cosmic-ray positrons in the early sixties (De Shong et al. 1964), many experiments using balloon-borne magnetic spectrometers have been carried out. It is generally believed that charged pions produced in the high energy collisions of cosmic-ray nucleons with the interstellar gas are the dominant source of $\mathrm{e}^{+}$via the decay chain $\pi^{+} \rightarrow \mu^{+} v_{\mu}$ and $\mu^{+} \rightarrow \mathrm{e}^{+} v_{\mathrm{e}} \bar{v}_{\mu}$. Based on this hypothesis, the expected fraction of $\mathrm{e}^{+}$in the framework of the Leaky Box Model appears to be consistent with the observations from $1 \mathrm{GeV}$ to about $10 \mathrm{GeV}$. However, above $10 \mathrm{GeV}$, some measurements indicate an excess of $\mathrm{e}^{+}$with respect to the standard predictions (see the summary in Golden et al. 1996). In order to

Send offprint requests to: C. Grimani,

e-mail: cgrimani@fis.uniurb.it

* Now at the Università di Urbino, Via S. Chiara, Urbino, Italy.

$\star \star$ Now at the Università dell'Aquila, Via Vetoio, L'Aquila, Italy.

$\star \star \star$ Deceased. explain these observations many speculations have been put forward. They include $\mathrm{e}^{+}$from the radioactive decay of ${ }^{56} \mathrm{Co}$ and accelerated in supernova remnants (Skibo \& Ramaty 1993), by the pair production in the magnetic fields of pulsars (Grimani 1996 and references therein), as end products of the annihilation of supersymmetric particles that were created at the beginning of the Universe (e.g. Tylka 1989) and from the interaction of high energy gamma rays with the ambient photons (e.g. Mastichiadis et al. 1991). It may be noted that except in the first case, equal amounts of $\mathrm{e}^{+}$and $\mathrm{e}^{-}$are produced in all the above processes. Although some recent experiments (Golden et al. 1996; Barwick et al. 1997; Coutu et al. 2001) do not support a large excess of $\mathrm{e}^{+}$above $10 \mathrm{GeV}$, additional measurements of the energy spectrum of $\mathrm{e}^{+}$are essential either to look for or to rule out these conjectured positron sources.

The large flux of $\mathrm{e}^{-}$with respect to $\mathrm{e}^{+}$in the primary cosmic radiation clearly indicates that most of the cosmic-ray electrons are not of secondary origin, but are produced in cosmicray sources. An accurate measurement of the energy spectra of 


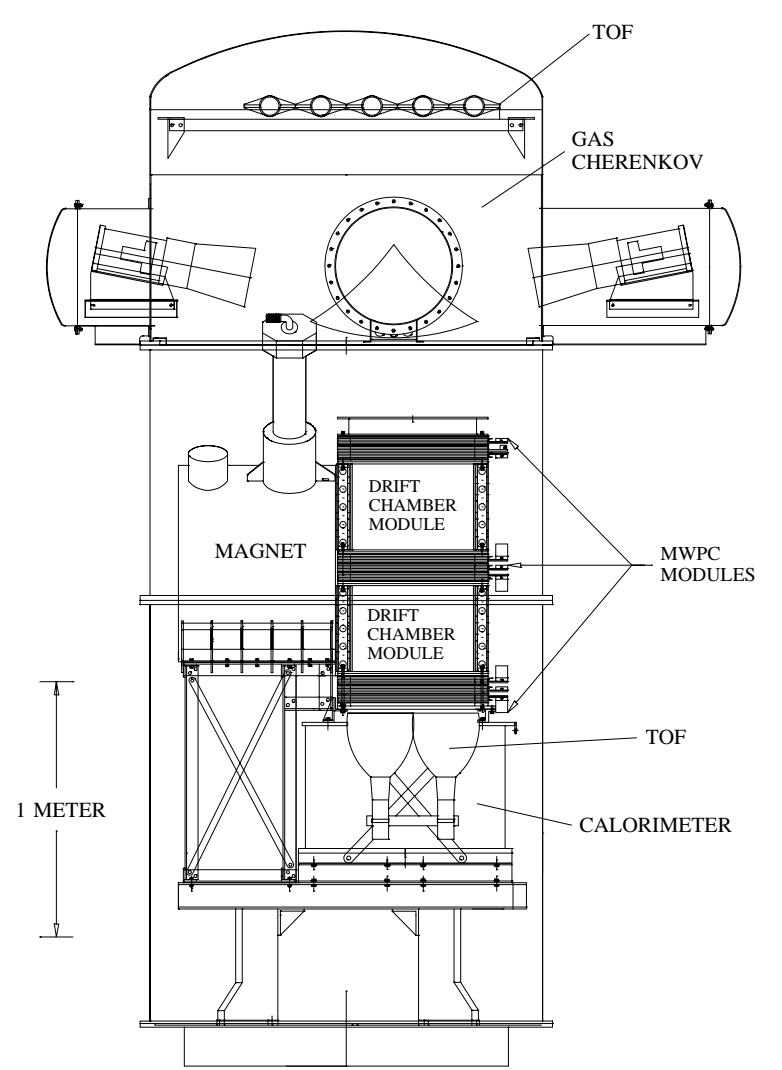

Fig. 1. Schematic view of the MASS-91 apparatus used in this measurement.

both $\mathrm{e}^{-}$and $\mathrm{e}^{+}$would provide an understanding of the spectral shape and the source of origin of the primary $\mathrm{e}^{-}$.

While $\mathrm{e}^{-}$can be identified by different experimental techniques, a magnet spectrometer is essential to distinguish between $\mathrm{e}^{-}$and $\mathrm{e}^{+}$. Many magnet spectrometer experiments have been carried out to study the electron component, but most of them measured the positron-to-electron ratio (Boezio et al. 1999 and references therein). Only 8 measurements in various energy intervals (Fanselow et al. 1969; Buffington et al. 1975; Golden et al. 1987; Golden et al. 1994; Barbiellini et al. 1997; Barwick et al. 1998; Boezio et al. 2000; Alcaraz et al. 2000) have been reported on the absolute spectrum of $\mathrm{e}^{+}$since 1969 . In this paper, a new measurement of the primary $\mathrm{e}^{+}$flux between 7 and $16 \mathrm{GeV}$, and electrons between 7 and $47 \mathrm{GeV}$ is presented. The data were collected by the balloon-borne instrument MASS-91 (Matter Antimatter Space Spectrometer) flown on September 23, 1991 from Fort Sumner, New Mexico. The results presented here are compared to the world data and to recent theoretical predictions.

\section{Instrument description}

A schematic diagram of the instrument used in this experiment is shown in Fig. 1. It consisted of a superconducting magnet spectrometer, a time-of-flight device (TOF), a gas Cherenkov counter and a streamer tube imaging calorimeter.

\subsection{Magnet spectrometer}

The magnet spectrometer consisted of a single coil superconducting magnet and a hybrid tracking system. The magnet was operated with a current of 120 A producing an inhomogeneous field with a maximum field strength in the region of tracking device of 2.2 Tesla, decreasing to 0.1 Tesla in the outer corners. The tracking system consisted of 2 modules of drift chambers (Hof et al. 1994) and 8 planes of multiwire proportional chambers (MWPC) adding up to a total height of $110 \mathrm{~cm}$ (Golden et al. 1991). Each drift chamber (DC) module consisted of 6 layers of hexagonal drift cells in the bending direction and 4 layers in the non-bending direction. The DC system was operated with $\mathrm{CO}_{2}$. The spatial resolution of the DC depended on the drift path. In this experiment, we achieved an average resolution better than $100 \mu \mathrm{m}$. The MWPCs were filled with magic gas and read out by a cathode coupled delay line system. Three MWPC layers were placed at the bottom of the tracking system, three were located between the two DC modules, and two layers were kept at the top of the tracking device. Four MWPCs were instrumented to read out both coordinates, and the rest measured positions in the bending direction. One MWPC chamber was not working during the flight. Both the MWPC and DC tracking systems together provided 19 position measurements along the direction of maximum curvature and 11 in the perpendicular direction. The spatial resolution of the MWPC varied between $200 \mu \mathrm{m}$ and $400 \mu \mathrm{m}$ in the bending direction and was about $1 \mathrm{~mm}$ in the non-bending direction. Because of the inhomogeneous magnetic field over the spectrometer volume, the deflection error distribution was found to peak at $4.75 \times 10^{-3} \mathrm{c} / \mathrm{GV}$, corresponding to $210 \mathrm{GV} / \mathrm{c}$ (Papini 1996). This rigidity value has been assumed as the maximum detectable rigidity (MDR). The present analysis has been limited to $40 \mathrm{GV} / \mathrm{c}$ for electrons and to $14 \mathrm{GV} / \mathrm{c}$ for positrons, well below the spectrometer MDR.

\subsection{Time-of-flight device}

The TOF device was made of two planes of scintillators separated by $2.36 \mathrm{~m}$. The upper one was at the top of the gondola and it consisted of two layers of scintillators, each segmented into 5 paddles of $20 \mathrm{~cm}$ width and variable lengths to match the round section of the cylindrical payload structure. Each paddle was viewed by a Hamamatsu R2490-01 phototube at the opposite end in each layer, so that the time difference between the signals from each layer can be made use of to locate the position of the particle passing through the paddle. The bottom plane was located just below the tracking device and had one layer of scintillator segmented into 2 paddles, viewed by phototubes at both ends. The coincidence between signals from the two planes provided the trigger for data acquisition. Signals from each paddle were independently digitized for the TOF measurement to determine the direction and velocity, and for the pulse-height analysis to obtain the ionization loss in the scintillators. With this configuration, a timing resolution of $370 \mathrm{ps}$ was achieved for singly charged particles. 


\subsection{Cherenkov detector}

The Cherenkov radiator (Golden et al. 1992) was located in the top section of the payload, which also housed the top scintillators. It was a $1 \mathrm{~m}$ tall cylinder isolated from the remaining gondola volume and was filled with Freon-12 at a pressure of 760 Torr at the ground. During the flight, there was a small change in the temperature and pressure, which resulted in a mean Cherenkov threshold of $\gamma_{\text {th }}=25.5$. The Cherenkov light was reflected and focused by 4 segmented spherical mirrors onto 4 BURLE C83073E phototubes. The response of the Cherenkov detector was position dependent due to the physical attributes of the mirror and the height, which reduced the path length of the particle traversing the radiator at the center. Ground muon data, taken prior to the flight, were used to create a two dimensional map of the mirror showing the signal amplitude. The detector performance was described elsewhere (Hof et al. 1996) and we used this information as a basis to determine the selection criteria for $\mathrm{e}^{+}$and $\mathrm{e}^{-}$.

\subsection{Imaging calorimeter}

The imaging calorimeter was located below the bottom scintillator. The calorimeter volume was filled with $50 \mathrm{~cm}$ long brass streamer tubes of cross-dimension $9 \times 7 \mathrm{~mm}^{2}$. There were 40 horizontal layers, each consisting of 64 streamer tubes. The tubes in alternate layers were arranged perpendicularly to each other in order to obtain a three dimensional view of an event passing through the calorimeter. The availability of a total of 2560 cells provided a very good granularity to examine the profile of the cascade development and interactions in the calorimeter (Codino et al. 1989). The total depth of the calorimeter was 7.3 radiation lengths, which corresponds to 0.7 inelastic interaction mean free path for protons.

This instrument was launched on September 23, 1991 at 8 am from Fort Sumner, New Mexico, where the geomagnetic threshold rigidity is about $4.5 \mathrm{GV} / \mathrm{c}$. The balloon reached the float altitude of $31.7 \mathrm{~km}$ at 10:12 am and after 9.8 hours the transition of the superconducting magnet occured, resulting in the loss of the magnetic field. The flight was later terminated and the mean atmospheric depth at the float altitude, during the period when the magnet was fully operational, was $5.8 \mathrm{~g} / \mathrm{cm}^{2}$.

\section{Analysis of the data}

We selected minimum ionizing singly charged particles for the analysis. The particle charge was determined using the $\mathrm{dE} / \mathrm{dx}$ information from the TOF paddles. We required that the particle deposited less than 1.8 times the most probable energy loss for minimum ionizing singly-charged particles in both the top and the bottom scintillators. These selected events were further subjected to various selection criteria in order to clearly distinguish the electron component from other minimum ionizing particles, such as protons and muons. These criteria are shown in Table 1 and are described below.
Table 1. Some parameters defining the electron and positron selection criteria in the spectrometer, Cherenkov counter and calorimeter.

\begin{tabular}{|c|c|}
\hline \multicolumn{2}{|l|}{ Spectrometer } \\
\hline $\begin{array}{l}\text { Position measurements in the } \\
\text { bending plane ( } x \text {-view) }\end{array}$ & $N_{\mathrm{ch}}(x) \geq 11$ \\
\hline $\begin{array}{l}\text { Position measurements in the } \\
\text { non-bending plane ( } y \text {-view) }\end{array}$ & $N_{\mathrm{ch}}(y) \geq 6$ \\
\hline$\chi^{2}$ values of the track ( $x$-view) & $\chi_{x}^{2} \leq 8$ \\
\hline$\chi^{2}$ values of the track ( $y$-view) & $\chi_{y}^{2} \leq 8$ \\
\hline Deflection error, $\sigma_{\eta}$ & $\sigma_{\eta} \leq 0.03(\mathrm{GV} / \mathrm{c})^{-1}$ \\
\hline Cherenkov counter & \\
\hline Signal amplitude for $\mathrm{e}^{+}$ & $\geq 8$ pe \\
\hline Signal amplitude for $\mathrm{e}^{-}$ & $\geq 0.5 \mathrm{pe}$ \\
\hline $\begin{array}{l}\text { Calorimeter } \\
\mathrm{e}^{-} \text {and } \mathrm{e}^{+}\end{array}$ & \\
\hline $\begin{array}{l}\text { Number of shower clusters, } n \text {, in } \\
\text { at least one view } \\
\mathrm{e}^{+} \text {only }\end{array}$ & $n \geq 4$ \\
\hline $\begin{array}{l}\text { Electromagnetic shower start, } z_{0} \text {, } \\
\text { in the }\end{array}$ & \\
\hline $\begin{array}{l}\text { first two calorimeter planes. } \\
\text { Opening angle of the }\end{array}$ & $z_{0} \leq 2$ \\
\hline electromagnetic cascade, $\theta$ & $\leq 25$ degrees \\
\hline
\end{tabular}

\subsection{Spectrometer selection}

In order to obtain a reliable information on the measured curvature, from which both the rigidity and the sign of charge can be determined, the following criteria were applied. In each event, there should be at least 11 position measurements out of the possible 19 positions in the bending direction and at least 6 out of 11 positions available in the non-bending direction. We also required the normalized $\chi^{2}$ in each view to be $\leq 8$. The error in the deflection measurement also needed to be $\leq 0.03$. After applying these criteria, negative curvature events were separated from the positive curvature events for further analysis to identify electrons and positrons from these samples.

\subsection{Cherenkov light requirement}

The major source of background for positrons comes from the interacting protons. Since positrons are expected to produce Cherenkov radiation in the plateau region, signal from the gas Cherenkov radiator plays an important role in discriminating positrons against protons. Therefore, we imposed a strict requirement of at least 8 photo-electrons (pe) to be recorded by the Cherenkov detector for $\mathrm{e}^{+}$. This criterion was relaxed to 0.5 photo-electron for $\mathrm{e}^{-}$only to ensure that the event had traversed the Cherenkov detector. It may be mentioned that no part of the Cherenkov detector was removed for the analysis. The $\mathrm{e}^{+}$selection being very strict would eliminate particles passing through certain sections of the Cherenkov detector, which produced low yield, leading to a lower selection efficiency. This resultant lower efficiency automatically takes care of the reduction of the geometrical factor due to the loss of effective area. 


\subsection{Calorimeter selection}

Finally, the calorimeter played a very important role in identifying electromagnetic cascades initiated by the electron component. The lateral spread of an electromagnetic shower is about 2 Molière radii, which is approximately $3.2 \mathrm{~cm}$ in our calorimeter. We defined shower cluster in a calorimeter plane, when at least 2 cells were activated within 5 cells from the particle trajectory. The particle trajectory in the calorimeter was obtained by extrapolating the trajectory measured by the spectrometer. We required a minimum of 4 shower clusters in at least one view. The main source of contamination for $\mathrm{e}^{-}$is the atmospheric muons and the above criterion rejected all the muons, as they do not suffer interactions like hadrons. In the case of $\mathrm{e}^{+}$we had to eliminate showers produced by proton interactions and therefore, we imposed two additional criteria for selecting $\mathrm{e}^{+}$. The first one was the requirement that the starting point of the shower should be within the first two calorimeter planes. This reduced the probability of proton interaction. The second was based on the measured electromagnetic shower characteristics, namely, that the opening angle of the cascade should be $\leq 25$ degrees. This criterion removed a large fraction of proton interactions, as they would have tracks coming out of the interaction vertex at large angles.

\subsection{Albedo particles}

In principle, an upward going $\mathrm{e}^{-}$would appear as a downward moving $\mathrm{e}^{+}$in the spectrometer. In this experiment, the TOF measurements can separate upward going particles from the downward going ones by more than 30 standard deviations. Indeed this criterion is redundant for electrons, as the requirement of an electromagnetic shower in the calorimeter alone makes the criterion for downward moving particle a very strict one. Thus, there is no contamination from albedo particles in the selected sample.

\subsection{The bar}

A $17 \mathrm{~kg}, 1.2 \mathrm{~m}$ long aluminium bar with a $7 \mathrm{~kg}$ steel swivel in the center was used to connect the gondola to the suspension line from the balloon. The particle loss by cascading and depletion of energy in this bar cannot be reliably estimated due to its peculiar shape, and hence we had rejected all events which came from the direction of this bar. This was carried out by extrapolating the tracks from the spectrometer to the location of the bar. By this procedure we were able to select a clean sample of events, which passed through similar amount of matter before being detected by the instrument and this procedure resulted in only a small reduction in the geometrical factor of about $10 \%$.

\subsection{Background estimates}

With the strict criteria used for selecting the $\mathrm{e}^{+}$using the Cherenkov counter and the calorimeter, the contamination by proton induced events was negligible in the sample of $\mathrm{e}^{+}$ selected in this analysis. In order to ensure this, selected samples of $500 \mathrm{e}^{-}$and $300 \mathrm{e}^{+}$events above $1 \mathrm{GV} / \mathrm{c}$ were visually scanned for non-interacting particles, representing either muons or protons, and for low multiplicity hadronic interactions. None was found and therefore, an upper limit of $1 \%$ at $95 \%$ confidence level was set for the background contamination on the selected $\mathrm{e}^{-}$and $\mathrm{e}^{+}$events (see Codino et al. 1997). This visual check also ensured that no spill-over protons contaminated the electrons in the highest energy interval chosen for the analysis.

It can also be noticed from Table 1 that we have applied looser criteria for $\mathrm{e}^{-}$compared to $\mathrm{e}^{+}$. These reflect the different background levels of the $\mathrm{e}^{+}$and $\mathrm{e}^{-}$. The ratio $\mathrm{p} / \mathrm{e}^{+}$in our energy range is about $10^{3}$ requiring a rejection factor for protons against $\mathrm{e}^{+}$to be at least a few times $10^{4}$. In the case of $\mathrm{e}^{-}$, the ratio $\mu^{-} / \mathrm{e}^{-}$at the float is about one requiring a rejection factor for muons against electrons of about 100, which is very easily achieved. Thus the above selection criteria provided a cleaner identification of the rarer $\mathrm{e}^{+}$, but with a slightly lower efficiency, and generated better statistics for $\mathrm{e}^{-}$.

With the above criteria, we selected for further analysis a total of 552 electrons between 5 and $40 \mathrm{GeV}$, and 37 positrons between 5 and $14 \mathrm{GeV}$. The selected events below the geomagnetic cut-off were analysed earlier (Codino et al. 1997).

\section{Determination of the flux and energy spectra of $\mathrm{e}^{+}$and $\mathrm{e}^{-}$}

The major parameters required to determine the flux of $\mathrm{e}^{+}$and $\mathrm{e}^{-}$are the detection efficiencies and geometrical factor. The procedures for evaluating these parameters have been reported earlier (Codino et al. 1997) and we describe them briefly below.

\subsection{Selection efficiencies}

Due to the position dependent response of time-of-flight scintillator counters to cosmic rays and the slightly higher setting of the discriminators, the particle trigger was not $100 \%$ efficient. An experiment was set up on the ground before the flight with a separate scintillator telescope to determine this efficiency and it was found that the trigger efficiency was $0.83 \pm 0.01$.

In order to determine the efficiency of detectors we selected samples of events using the remaining devices and applied the selection criteria of the detector in question. A sample of 20000 protons gathered during the flight, which did not interact in the calorimeter, was used to determine the scintillator selection efficiency and found to be $0.91 \pm 0.01$.

The efficiency of the spectrometer was determined using a two-step procedure. The TOF system was first used to select events with $Z=1$ and $\beta=1$. The calorimeter was then used to select electromagnetic showers, whose extrapolated trajectories passed through the active volume of the tracking system. These selected events were then fitted with the tracking algorithm and were subjected to the standard criteria given in Table 1 . The efficiency was $0.94 \pm 0.01$.

In order to get the efficiency resulting from the two selection criteria used in the Cherenkov counter for $\mathrm{e}^{+}$and $\mathrm{e}^{-}$, a sample of $\mathrm{e}^{-}$were selected using the calorimeter. These electron 
Table 2. Detector efficiencies for electrons and positrons.

\begin{tabular}{|c|c|c|}
\hline Detector & Efficiency & Remarks \\
\hline Spectrometer & $0.94 \pm 0.01$ & for $\mathrm{e}^{-}$and $\mathrm{e}^{+}$ \\
\hline \multirow[t]{2}{*}{ Calorimeter } & $0.98 \pm 0.01$ & for $\mathrm{e}^{-}$ \\
\hline & $0.96 \pm 0.01$ & for $\mathrm{e}^{+}$ \\
\hline Scintillator counter system & $0.91 \pm 0.01$ & for $\mathrm{e}^{-}$and $\mathrm{e}^{+}$ \\
\hline \multirow[t]{2}{*}{ Cherenkov counter } & $0.98 \pm 0.01$ & for $\mathrm{e}^{-}$ \\
\hline & $0.84 \pm 0.01$ & for $\mathrm{e}^{+}$ \\
\hline
\end{tabular}

events were visually examined to make sure that the the sample was pure and they were expected to radiate in the plateau region. We then applied the two different selection criteria for the Cherenkov counter obtaining an efficiency of $0.98 \pm 0.01$ for $\mathrm{e}^{-}$ and $0.84 \pm 0.01$ for $\mathrm{e}^{+}$. The lower efficiency for the $\mathrm{e}^{+}$criterion is indeed reflecting the loss of regions in the mirror, which were found to produce smaller number of photo-electrons.

The calorimeter efficiency to record a single track is $100 \%$. We have examined 293 electromagnetic showers visually and found that the efficiency was only $0.98 \pm 0.01$ when applying the requirement of a minimum of 4 shower clusters in at least one view of the calorimeter. In the case of $\mathrm{e}^{+}$selection criteria, the efficiency was reduced from electron efficiency only by another $2 \%$.

All these detector selection efficiencies are summarized in Table 2.

\subsection{Geometrical factor}

The geometrical factor was calculated by analytical integration using the actual magnetic field configuration and by incorporating the geometrical acceptance employed in the analysis procedure. We also rejected those parts of the solid angle in which the trajectories passed through the bar. The calculated geometrical factor was found to vary below $1 \mathrm{GV} / \mathrm{c}$ (Codino et al. 1997). It remained constant and equal to $182 \pm 1 \mathrm{~cm}^{2} \mathrm{sr}$ above this rigidity for both positrons and electrons. The total flight time used in this analysis is $35330 \pm 10 \mathrm{~s}$, out of which the dead time fraction of the instrument was 0.36 .

\subsection{Energy spectra determination}

In Col. 3 of Tables 3 and 4 we show for positrons and electrons, respectively, the number of events, which survived the $\mathrm{e}^{+}$and $\mathrm{e}^{-}$selection criteria described above.

The two rigidities defining each bin are those measured at the spectrometer. The fluxes at the spectrometer (SPEC) were obtained by dividing the observed number of particles in each rigidity bin by the efficiencies, geometrical factor and total collection time. These differential fluxes are shown in Col. 4. Since the rigidity and energy are nearly the same for these light leptons, the energy interval is identical to the rigidity interval. From a study of the effect of the spectrometer resolution functions (Papini 1996) we inferred that the energy spectrum is not distorted by rigidity measurements in the energy interval of observation of both $\mathrm{e}^{+}$and $\mathrm{e}^{-}$, since it is well below the
Table 3. Positron observation summary.

\begin{tabular}{lllll}
\hline \hline $\begin{array}{l}\text { Rigidity } \\
(\mathrm{GV} / \mathrm{c})\end{array}$ & $\begin{array}{l}\text { Energy } \\
(\mathrm{GeV})\end{array}$ & $\begin{array}{l}\text { Number } \\
\text { of } \\
\text { TOA }\end{array}$ & $\begin{array}{l}\text { Flux } \\
\left(\mathrm{m}^{2} \mathrm{srs} \mathrm{GeV}\right)^{-1}\end{array}$ & $\begin{array}{l}\text { Flux } \\
\left(\mathrm{m}^{2} \text { sr s GeV }\right)^{-1}\end{array}$ \\
\hline $5-6$ & 7.51 & 9 & $0.038 \pm 0.013$ & $0.020 \pm 0.009$ \\
$6-8$ & 9.48 & 12 & $0.025 \pm 0.008$ & $0.014 \pm 0.006$ \\
$8-10$ & 12.24 & 9 & $0.019 \pm 0.006$ & $0.011 \pm 0.004$ \\
$10-14$ & 15.98 & 7 & $0.007 \pm 0.003$ & $0.0038 \pm 0.0017$ \\
\hline
\end{tabular}

Table 4. Electron observation summary.

\begin{tabular}{|c|c|c|c|c|}
\hline $\begin{array}{l}\text { Rigidity } \\
(\mathrm{GV} / \mathrm{c})\end{array}$ & $\begin{array}{l}\text { Energy } \\
(\mathrm{GeV}) \\
\text { TOA }\end{array}$ & $\begin{array}{l}\text { Number } \\
\text { of } \\
\text { events }\end{array}$ & $\begin{array}{l}\text { Flux } \\
\left(\mathrm{m}^{2} \mathrm{sr} \mathrm{s} \mathrm{GeV}\right)^{-1} \\
\text { SPEC }\end{array}$ & $\begin{array}{l}\text { Flux } \\
\left(\mathrm{m}^{2} \mathrm{sr} \mathrm{s} \mathrm{GeV}\right)^{-1} \\
\text { TOA }\end{array}$ \\
\hline $5-6$ & 7.51 & 157 & $0.567 \pm 0.047$ & $0.404 \pm 0.034$ \\
\hline $6-7$ & 8.88 & 85 & $0.307 \pm 0.034$ & $0.207 \pm 0.023$ \\
\hline $7-8$ & 10.24 & 73 & $0.261 \pm 0.031$ & $0.169 \pm 0.020$ \\
\hline $8-9$ & 11.61 & 47 & $0.168 \pm 0.025$ & $0.106 \pm 0.016$ \\
\hline $9-10$ & 12.98 & 34 & $0.122 \pm 0.021$ & $0.077 \pm 0.013$ \\
\hline $10-12$ & 14.95 & 48 & $0.085 \pm 0.012$ & $0.054 \pm 0.008$ \\
\hline $12-14$ & 17.71 & 36 & $0.064 \pm 0.011$ & $0.040 \pm 0.007$ \\
\hline $14-16$ & 20.46 & 18 & $0.032 \pm 0.008$ & $0.020 \pm 0.005$ \\
\hline $16-20$ & 24.34 & 19 & $0.017 \pm 0.004$ & $0.011 \pm 0.002$ \\
\hline $20-25$ & 30.42 & 16 & $0.011 \pm 0.003$ & $0.0071 \pm 0.0018$ \\
\hline $25-30$ & 37.34 & 10 & $0.0071 \pm 0.0022$ & $0.0045 \pm 0.0014$ \\
\hline $30-40$ & 46.92 & 9 & $0.0032 \pm 0.0011$ & $0.0020 \pm 0.0007$ \\
\hline
\end{tabular}

MDR. The determination of the energy spectra of $\mathrm{e}^{+}$and $\mathrm{e}^{-}$at the top of the atmosphere (TOA) requires important corrections to the corresponding spectra observed at the instrument. They include (1) extrapolation of the spectrum to the float altitude after correcting for the Bremsstrahlung and ionization energy losses above the spectrometer, (2) subtraction of the $\mathrm{e}^{+}$and $\mathrm{e}^{-}$ produced in the overlying atmosphere and (3) extrapolation of the resultant spectrum to the top of the atmosphere. These are briefly described below and the details are given in our earlier publications (Golden et al. 1994, 1996; Codino et al. 1997).

The average thickness of material traversed by particles in the detector before they arrive at the spectrometer is 0.13 radiation length, including the gondola shell. From the energy spectra at the spectrometer we derived the spectra of both $\mathrm{e}^{+}$and $\mathrm{e}^{-}$at the top of the payload by applying the cascade equation describing the energy loss of electrons due to Bremsstrahlung and ionization; the corresponding shift in the particle energy has been also determined. These spectra at the float altitude contain both the energy degraded primary particles and the secondary $\mathrm{e}^{+}$and $\mathrm{e}^{-}$produced in the overlying atmosphere of $5.8 \mathrm{~g} / \mathrm{cm}^{2}$. The contribution of secondary $\mathrm{e}^{+}$and $\mathrm{e}^{-}$at the float altitude was estimated from Stephens (1981) and subtracted from the measured spectra at float.

The residual spectra thus obtained at float contain not only the energy degraded primary electron component, but also a few secondaries produced by the electromagnetic cascading process, whose contribution to the $\mathrm{e}^{+}$is non-trivial. Therefore, the extrapolation of the residual spectra to the top of the atmosphere was carried out by simultaneously solving the cascade 
Table 5. Positron fraction.

\begin{tabular}{ll}
\hline \hline $\begin{array}{l}\text { Energy } \\
(\mathrm{GeV})\end{array}$ & $\frac{\mathrm{e}^{+}}{\mathrm{e}^{+}+\mathrm{e}^{-}}$ \\
\hline 7.51 & $0.048 \pm 0.020$ \\
9.48 & $0.070 \pm 0.027$ \\
12.24 & $0.107 \pm 0.037$ \\
15.98 & $0.076 \pm 0.032$ \\
\hline
\end{tabular}

equations for $\mathrm{e}^{+}, \mathrm{e}^{-}$and $\gamma$-rays, by including Bremsstrahlung, pair production and ionization processes. The energy shift resulting from the energy loss was used to derive the flux values of $\mathrm{e}^{+}$and $\mathrm{e}^{-}$at the top of the atmosphere. These flux values are given in Col. 5 of Tables 3 and 4. The median energy in each bin at the top of the atmosphere is also shown in Col. 2 of Tables 3 and 4 . The fraction of positrons in the cosmic radiation, $\left[\mathrm{e}^{+} /\left(\mathrm{e}^{+}+\mathrm{e}^{-}\right)\right]$, is shown in Table 5 .

\section{Results and discussion}

\subsection{Electron spectrum}

The differential flux values of electrons measured by this experiment at the top of the atmosphere are plotted in Fig. 2 by solid circles in the energy region from 7 to $47 \mathrm{GeV}$.

It can be seen from this figure that the energy spectrum can be fitted by a simple power-law in the above energy domain with a spectral index $\beta\left(\mathrm{e}^{-}\right)=-2.89 \pm 0.10$.

We have also plotted in this figure the electron spectra measured by magnet spectrometers during the last two decades. There is a general agreement among all these experiments in the energy region covered by this experiment. Notice that the flux values of Golden et al. (1984), shown as open squares, were given for the interstellar space (ISM) and hence the spectrum appears to be steeper than the rest of the data. The present results are consistent with the results from an earlier flight with the same apparatus (except the tracking system), which are shown as open circles in this figure (Golden et al. 1994). This experiment was carried out from Prince Albert, Saskatchewan (Canada) in 1989 at a lower rigidity cut-off of $0.650 \mathrm{GV} / \mathrm{c}$, which extends the spectrum to lower energies. It can also be seen that the AMS spectrum (Alcaraz et al. 2000) seems to deviate from other results below $2 \mathrm{GeV}$.

For a comparison, we have included in Fig. 2 the recent theorethical calculations of the expected electron spectrum by Stephens (2001a,b), who used a plerion type injection spectrum. The spectrum shown by the dashed curve is for the ISM and that shown by the solid curve is at the Earth with a solar modulation parameter $\phi=0.55 \mathrm{GV}$, which was used by him to match the results of Boezio et al. (2000). The dotted curve is the calculated spectrum by Moskalenko \& Strong (M\&S 1998) in the ISM and we have modulated their spectrum using the spherically symmetric model in the force-field approximation (Gleeson \& Axford 1968) with the same modulation parameter as in the case of Stephens. We have used the M\&S results based on the propagation without re-acceleration. Despite different assumptions made by these authors both in the nature of

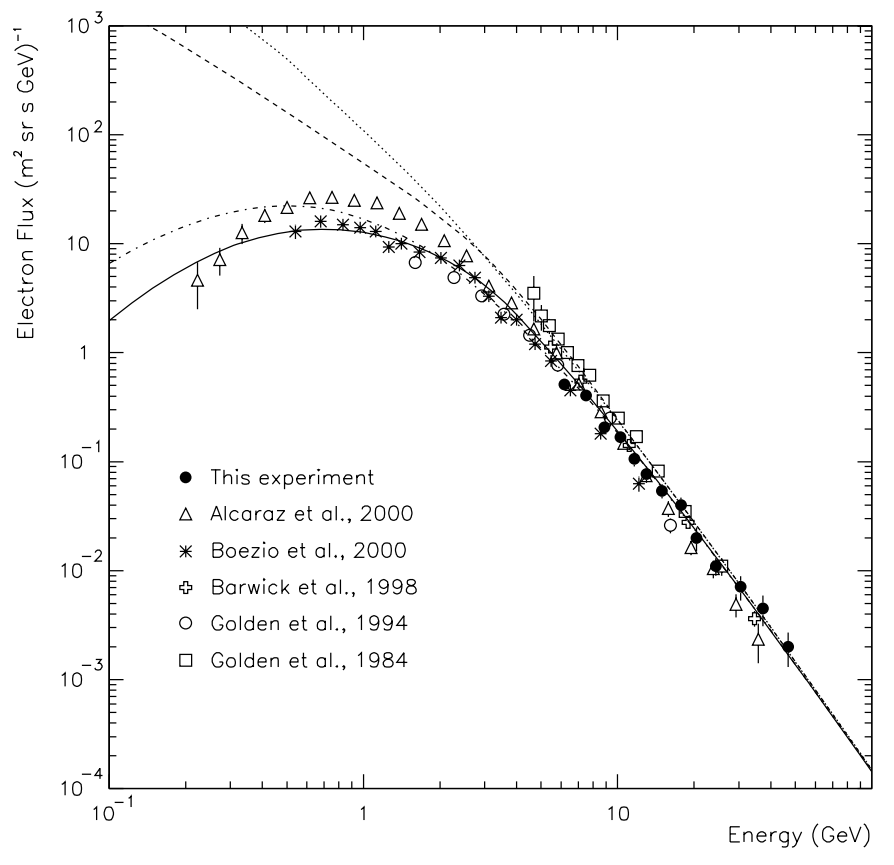

Fig. 2. Electron energy spectrum measured by MASS-91 (solid circles). Results from other experiments published in the last twenty years are also shown. Data have been compared to theoretical calculations in the interstellar medium (dotted curve: Moskalenko \& Strong 1998; dashed curve: Stephens 2001a,b) and at the Earth by considering the effect of the solar modulation using a modulation parameter $\phi=0.55$ (dot-dashed curve: Moskalenko \& Strong 1998; solid curve: Stephens 2001a,b)

the injection spectrum and the propagation parameters, there is a good agreement between these two calculations at energies above $1 \mathrm{GeV}$. These calculations also agree remarkably well with the experimental data. The apparent difference exhibited at lower energies can be tested in the future with space experiments.

\subsection{Positron spectrum}

The positron flux values obtained in this experiment are shown in Fig. 3 by solid circles. Symbols used in this and the following figures are the same as in Fig. 2. Because of the limited data points from this experiment, we have made use of the available data above $7 \mathrm{GeV}$ to determine the spectral index of the energy spectrum in this energy region. The spectral index thus obtained is $\beta\left(\mathrm{e}^{+}\right)=-3.37 \pm 0.26$. As in the case of electrons, we have included in this figure the data from experiments carried out during the last two decades. There is a general agreement among all the experiments. The existing errors are rather large and it is very essential to extend the measurements of the absolute spectrum of positrons both at low and at high energies.

We have also shown in this figure the theoretical calculations of Stephens as well as those of Moskalenko and Strong; both have estimated the equilibrium spectrum of positrons in the Galaxy resulting from the interactions of cosmic-ray nucleons with the interstellar gas. As in the case of Stephens, we have chosen the estimate of positron spectrum by $M \& S$ without re-acceleration and produced by cosmic-ray nuclei having 


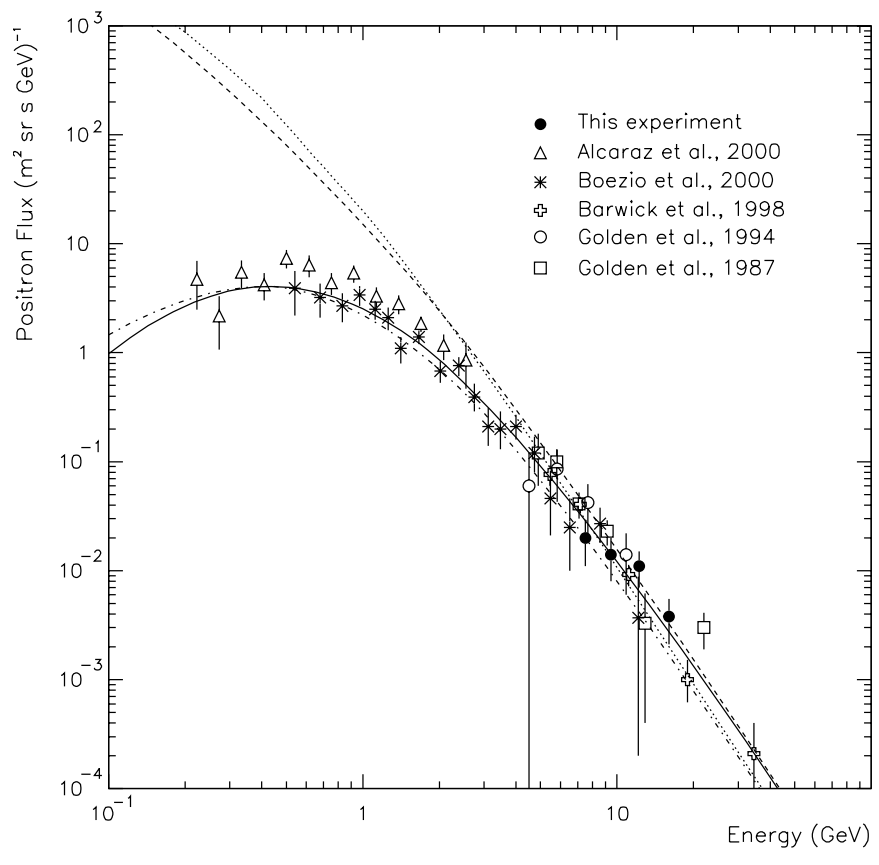

Fig. 3. Positron energy spectrum measured by MASS-91 (solid circles) and previous experiments. Theoretical curves have the same meaning as in Fig. 2.

an asymptotic spectral shape as that observed near the Earth. It can be seen that the estimated spectrum of M\&S is steeper than that of Stephens. As a result, while Stephens' estimate is in good agreement with the data, $M \& S$ estimate falls at the lower end of the data envelope above a few GeV.

\subsection{Fraction of positrons in the electron component}

The positron ratio defined as the fraction of positrons in the electron component, $R=\mathrm{e}^{+} /\left(\mathrm{e}^{+}+\mathrm{e}^{-}\right)$is shown in Fig. 4 along with other existing data obtained during the last two decades using magnet spectrometers. Though our results with 4 data points appear to show an increase with energy over the limited energy range, they are quite consistent with a constant ratio above $7 \mathrm{GeV}$. From this figure it is possible to notice that the value of $R$ spreads over a band between 0.025 and 0.15 in the energy interval $7-30 \mathrm{GeV}$, with an average value of $0.064 \pm 0.003$. It also can be seen from the data that while the observed intensity of $\mathrm{e}^{+}$is less than $10 \%$ of the electron component of the cosmic radiation above $2 \mathrm{GeV}$, they constitute about $30 \%$ below about $0.3 \mathrm{GeV}$. The rapid decrease of $R$ noticed at lower energies seems to suddenly change around $2 \mathrm{GeV}$. Though there is a large spread in the data above a few $\mathrm{GeV}$, the general trend appears to suggest that the ratio tends to become nearly constant or might even increase slowly.

Theoretical estimates of the ratio are shown in this figure for comparison. Among these, the calculated ratio by Stephens (solid curve at Earth) is in good agreement with the data especially below $3 \mathrm{GeV}$ and consistent with the general trend in the high energy data within the uncertainties of the measurements. However, the M\&S calculations (thin dash-dotted curve) do not fit the data over the entire energy region. At the same time,

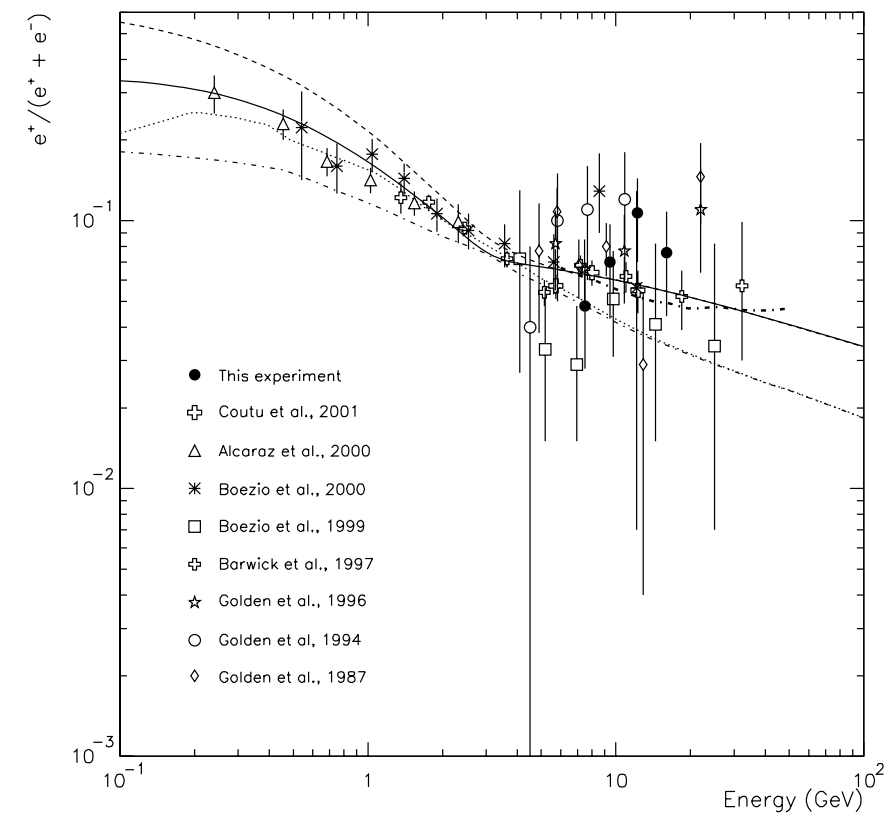

Fig. 4. Positron fraction versus energy. Solid circles represent the MASS-91 measurements. Data published in the last twenty years has also been reported. Theoretical curves have been estimated from Moskalenko \& Strong (1998) (dotted curve in the interstellar medium, dot-dashed curve near the Earth by considering a modulation parameter $\phi=0.55 \mathrm{GV})$ and Stephens $(2001 \mathrm{a}, \mathrm{b})$ (dashed curve in the interstellar medium, solid curve near the Earth by considering a modulation parameter $\phi=0.55 \mathrm{GV}$ ). The thick dot-dashed curve shows the expected positron fraction near the Earth when a positron component generated at the pulsar polar cap is added to the Moskalenko and Strong calculations.

it is also clear from the figure that the ratio should decrease with energy, based on the secondary hypothesis, specially at high energies. If the ratio either becomes constant or increases above a few $\mathrm{GeV}$, it is essential to invoke the electron-positron pair production process at the pulsar polar cap (Grimani 1996) along with the secondary production in the ISM. The heavy dashdotted curve in this figure is an example shown by combining the estimated pulsar contribution, assuming a pulsar birthrate of one in 60 years, from Grimani with the M\&S curve. Any increase of the ratio can also be explained by the annihilation of supersymmetric particles, but this enhancement would vanish at energies above the mass of such particles (Grimani 2000). It is clear that each one of the above hypotheses has a distinct prediction at high energies, which can be easily tested when measurements could extend to energies of at least $100 \mathrm{GeV}$.

Acknowledgements. This work was supported by NASA, Grant NAG110, the National Scientific Balloon Facility, USA, the Istituto Nazionale di Fisica Nucleare (INFN), the Agenzia Spaziale Italiana, Italy and the German Space Agency, DARA, Germany.

We thank Mirko Boezio of the Istituto Nazionale di Fisica Nucleare, Trieste, Italy for useful communications.

\section{References}

Alcaraz, J., Alpat, B., Ambrosi, G., et al. 2000, Phys. Lett. B, 484, 10 Barbiellini, G., Basini, G., Bellotti, R., et al. 1996, A\&A, 309, L15 
Barbiellini, G., Basini, G., Bellotti, R., et al. 1997, Proc. 25th Int. Cosmic Ray Conf., Durban, 4, 221

Barwick, S. W., Beatty, J. J., Bhattacharyya, A., et al. 1997, ApJ, 482, L191

Barwick, S. W., Beatty, J. J., Bower, C. R., et al. 1998, ApJ, 498, 779

Boezio, M., Ambriola, M. L., Barbiellini, G., et al. 1999, Proc. 26th Int. Cosmic Ray Conf., Salt Lake City, 3, 57

Boezio, M., Carlson, P., Francke, T., et al. 2000, ApJ, 532, 653

Buffington, A., Orth, C. D., \& Smoot, G. F. 1975, ApJ, 199, 669

Codino, A., Federico, C., Grimani, C., et al. 1989, Il Nuovo Cimento B, 103, 319

Codino, A., Brunetti, M. T., Federico, C., et al. 1997, J. Phys. G, 23, 1751

Coutu, S., Beach, A. S., Beatty, J. J., et al. 2001, Proc. 27th Int. Cosmic Ray Conf., Hamburg, 5, 1687

De Shong, J. A., Hildebrand, R. H., \& Meyer, P. 1964, Phys. Rev. Lett., 12,3

Fanselow, J. L., Hartman, R. C., Hildebrad, R. H., \& Meyer, P. 1969, ApJ, 158, 771

Gleeson, L. J., \& Axford, W. I. 1968, ApJ, 154, 1011

Golden, R. L., Mauger, B. G., Badhwar, G. D., et al. 1984, ApJ, 287, 622

Golden, R. L., Mauger, B. G., Horan, S., et al. 1987, A\&A, 188, 145

Golden, R. L., Grimani, C., Hull, R., et al. 1991, Nucl. Instr. Meth., A306, 366
Golden, R. L., Grimani, C., Kimbell, B. L., et al. 1992, PAL Tech. Note, No. 224, available on request

Golden, R. L., Grimani, C., Kimbell, B. L., et al. 1994, ApJ, 436, 769

Golden, R. L., Stochaj, S. J., Stephens, S. A., et al. 1996, ApJ, 457, 103

Grimani, C. 1996, Ap\&SS, 241, 295

Grimani, C. 2000, Nucl. Phys. B, Proc. Suppl., 85, 298

Harding, A., \& Ramaty, R. 1987, Proc. 20th Int. Cosmic Ray Conf., Moscow, 2, 92

Hof, M., Bremgrich, M., Menn, W., et al. 1994, Nucl. Instr. Meth., A345, 561

Hof, M., Menn, W., Pfeifer, C., et al. 1996, ApJ, 467, L33

Mastichiadis, M., Protheroe, R. J., \& Stephens, S. A. 1991, Proc. Astron. Soc. Aust., 9, 115; Proc. 22nd Int. Cosmic Ray Conf., Dublin, 2, 145

Moskalenko, I. V., \& Strong, A. W. 1998, ApJ, 493, 694

Papini, P. 1996, Ph.D. Thesis, University of Florence, Italy

Skibo, S. G., \& Ramaty, R. 1993, Proc. 23rd Int. Cosmic Ray Conf., Calgary, 2, 132

Stephens, S. A. 1981, Proc. 17th Int. Cosmic Ray Conf., Paris, 4, 282

Stephens, S. A. 2001a, in Origin and acceleration of cosmic rays, ed. M. Israel, Adv. Sp. Res., 27(4), 687

Stephens, S. A. 2001b, Proc. 27th Int. Cosmic Ray Conf., Hamburg, 5,1799

Tylka, A. J. 1989, Phys. Rev. Lett., 63, 840 